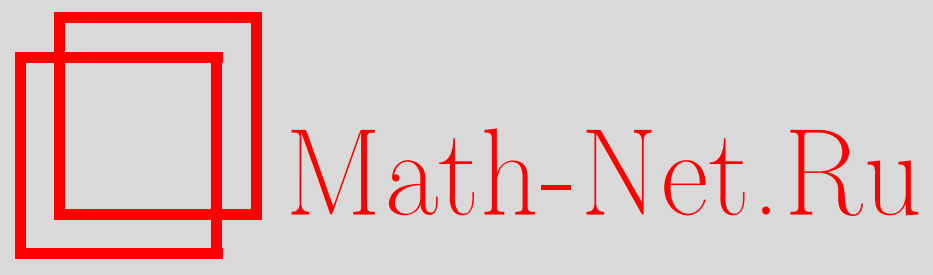

Г. С. Маканин, Т. А. Маканина, Параметризация решений некоторых уравнений квадратов в свободном моноиде, Дискрет. матем., 1999, том 11, выпуск 3, 133-148

DOI: https://doi.org/10.4213/dm384

Использование Общероссийского математического портала Math-Net.Ru подразумевает, что вы прочитали и согласны с пользовательским соглашением http: //www.mathnet.ru/rus/agreement

Параметры загрузки:

IP: 3.81 .55 .215

26 апреля 2023 г., $17: 23: 34$

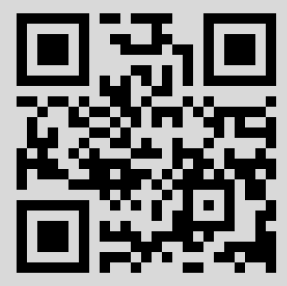


УДК 519.4

\section{Параметризация решений некоторых уравнений квадратов в свободном моноиде}

(C) 1999 г. . С. Маканин, Т. А. Маканина

Вводятся параметризующие функции $F a, L a, D o$, зависящие от словарных переменных, натуральных переменных и переменных, значениями которых являются конечные последовательности натуральных переменных. C помощью параметризующих функций $F a, L a, D o$ описываются конечные формулы для семейства решений уравнения

$$
x_{1}^{2} x_{2}^{2} x_{3}^{2}=x_{4}^{2}
$$

в свободном моноиде.

Работа выполнена при поддержке Российского фонда фундаментальных исследований, проект 98-01-00885.

\section{1. Введение}

Параметризовать решения уравнения в свободном моноиде - это значит выписать общее решение (множество всех решений) этого уравнения при помощи конечных формул, зависящих от параметров. В 1967 году Хмелевский [1] показал, что множество всех решений уравнения с тремя неизвестными в свободном моноиде может быть параметризовано конечным числом формул, зависящих от словарных переменных и натуральных переменных. Такая параметризация теперь называется примитивной. В 1971 году Хмелевский [2] доказал, что множество всех решений уравнения Маркова

$$
x_{1} x_{2} x_{3}=x_{3} x_{4} x_{1}
$$

в свободном моноиде не допускает примитивной параметризации.

В дальнейшем [3-5] для параметризации решений уравнения в свободном моноиде стали использовать параметризующие функции, зависящие от словарных переменных, натуральных переменных и переменных, значениями которых являются конечные последовательности натуральных переменных.

При исследовании структуры решения произвольного уравнения в свободном моноиде важную роль играют уравнения квадратов, то есть уравнения вида

$$
x_{1}^{2} x_{2}^{2} \ldots x_{r}^{2}=x_{r+1}^{2} x_{r+2}^{2} \ldots x_{n}^{2} .
$$

В настоящей статье исследуются три уравнения квадратов в свободном моноиде. Доказано, что уравнения

$$
x_{1}^{2} x_{2}^{2}=x_{3}^{2}
$$


и

$$
x_{1}^{2} x_{2}^{2}=x_{3}^{2} x_{4}^{2}
$$

допускают примитивную параметризацию своих решений. Для параметризации решений уравнения

$$
x_{1}^{2} x_{2}^{2} x_{3}^{2}=x_{4}^{2}
$$

в свободном моноиде в статье определены рекурсивные функции

$$
\begin{aligned}
& { }^{F a}\left(x_{1}, x_{2}, x_{3}\right)_{i}^{\lambda_{1}, \ldots, \lambda_{s}}, \quad s \geqslant 0, \quad i=1,2, \\
& { }^{L a}\left(x_{1}, x_{2}, x_{3}, x_{4}\right)_{i}^{\mu_{1}, \ldots, \mu_{s}}, \quad s \geqslant 0, \quad i=1,2,3,4 \text {, } \\
& { }^{D o}\left(x_{1}, x_{2}, x_{3}, x_{4}\right)_{i}^{\nu_{1}, \ldots, \nu_{s}}, \quad s \geqslant 0, \quad i=1,2,3,4 \text {, }
\end{aligned}
$$

зависящие от словарных переменных $x_{i}$ и рекурсивно от конечных последовательностей: натуральных переменных $\lambda_{i}$, переменных $\mu_{i}$ для последовательностей переменных $\lambda_{i}$ и переменных $\nu_{i}$ для последовательностей переменных $\mu_{i}$. Показано, каким образом множество всех решений уравнения

$$
x_{1}^{2} x_{2}^{2} x_{3}^{2}=x_{4}^{2}
$$

в свободном моноиде параметризуется конечными формулами, построенными при помощи параметризующих функций $F a, L a, D o$.

\section{2. Определения и обозначения}

Пусть П - свободный моноид (свободная полугруппа с единицей) со счетным алфавитом образующих

$$
a_{1}, a_{2}, \ldots, a_{k}, \ldots
$$

Пусть

$$
x_{1}, x_{2}, \ldots, x_{n}
$$

- алфавит словарных переменных.

Уравнение в свободном моноиде задается равенством слов $\Phi=\Psi$ в алфавите $(1),(2)$. Слова в алфавите (1), входящие в уравнение $\Phi=\Psi$, называются коэффициентами уравнения. Если $\Phi, \Psi$ - пустые слова, то уравнение $\Phi=\Psi$ называется тривиальным уравнением.

Преобразование

$$
T=\left\{\begin{array}{c}
x_{1} \rightarrow X_{1}, \\
\ldots \\
x_{n} \rightarrow X_{n},
\end{array}\right.
$$

где $X_{1}, \ldots, X_{n}$ - слова в алфавите (1), называется решением уравнения $\Phi=\Psi$, если в результате применения этого преобразования и последующих сокращений мы получаем тривиальное уравнение. Объединяя преобразования переменных фигурной скобкой, мы указываем, что в данном преобразовании замена переменных происходит одновременно. 
В этой работе мы рассматриваем бескоэффициентные уравнения в свободном моноиде. Длина слова $A$ в алфавите (1) обозначается через $|A|$. Пустое слово обозначается через 1 .

Пусть

$$
\lambda_{1}, \lambda_{2}, \ldots, \lambda_{t}, \ldots
$$

- счетный алфавит переменных натуральных чисел (называемых также натуральными параметрами).

Любое слово в алфавите $x_{1}, x_{2}, \ldots, x_{n}$ является примитивным параметрическим словом. Если $P$ - примитивное параметрическое слово, $\lambda$ - натуральный параметр, то $(P)^{\lambda}$ является примитивным параметрическим словом. Если $P, Q$ - примитивные параметрические слова, то $P Q$ является примитивным параметрическим словом.

Преобразование переменных вида

$$
\left\{\begin{aligned}
x_{1} & \rightarrow X_{1} \\
& \cdots \\
x_{n} & \rightarrow X_{n}
\end{aligned}\right.
$$

где каждое слово $X_{i}$ есть слово в алфавите (1), называется коэффициентным преобразованием.

Преобразование переменных вида

$$
\left\{\begin{aligned}
x_{1} & \rightarrow W_{1}\left(x_{1}, \ldots, x_{n}, \lambda_{1}, \ldots, \lambda_{t}\right), \\
& \ldots \\
x_{n} & \rightarrow W_{n}\left(x_{1}, \ldots, x_{n}, \lambda_{1}, \ldots, \lambda_{t}\right),
\end{aligned}\right.
$$

где каждое $W_{i}$ есть примитивное параметрическое слово, называется примитивным параметрическим преобразованием. Неподвижные переменные часто не выписываются.

Примитивное параметрическое преобразование (коэффициентное преобразование) называется примитивным параметрическим решением (решением) уравнения $\Phi=\Psi$ в свободном моноиде, если в результате применения этого преобразования к уравнению $\Phi=\Psi$ и последующих сокращений мы получаем тривиальное уравнение.

Любая натуральная переменная является комплексной натуральной переменной. Переменная, значениями которой являются конечные последовательности комплексных натуральных переменных, является комплексной натуральной переменной.

Любое примитивное параметрическое слово является параметризующей функцией. Если для всякой последовательности комплексных натуральных переменных $\omega_{1}, \ldots, \omega_{k}$ из заданного класса последовательностей комплексных натуральных переменных выражение $F\left(x_{1}, \ldots, x_{n}, \omega_{1}, \ldots, \omega_{k}\right)$ является суперпозицией параметризующих функций, то выражение $F\left(x_{1}, \ldots, x_{n}, \omega\right)$, где $\omega-$ комплексная натуральная переменная, значением которой являются последовательности $\omega_{1}, \ldots, \omega_{k}$, является параметризующей функцией.

Суперпозиция параметризующих функций называется параметрическим словом.

Преобразование переменных вида

$$
\left\{\begin{aligned}
x_{1} & \rightarrow F_{1}\left(x_{1}, \ldots, x_{n}, \omega_{1}, \ldots, \omega_{m}\right), \\
& \ldots \\
x_{n} & \rightarrow F_{n}\left(x_{1}, \ldots, x_{n}, \omega_{1}, \ldots, \omega_{m}\right),
\end{aligned}\right.
$$


где каждое $F_{i}$ есть параметрическое слово, называется параметрическим преобразованием.

Говорят, что параметрическое преобразование $T$ содержит коэффициентное преобразование $C$ посредством вспомогательного преобразования $I$, если $T I=C$.

Конечный список параметрических решений уравнения $E$ будем называть общим решением уравнения $E$, если каждое решение уравнения $E$ содержится в некотором параметрическом решении этого списка. Общее решение уравнения $E$ обозначается через $\langle E\rangle$.

Уравнение в свободном моноиде часто рассматривается вместе с условиями на натуральные параметры и на длины словарных переменных.

Условие на натуральные параметры имеет вид

$$
L_{1}\left(\lambda_{1}, \ldots, \lambda_{t}\right) \leqslant L_{2}\left(\lambda_{1}, \ldots, \lambda_{t}\right)
$$

где $L_{1}, L_{2}$ - линейные полиномы от натуральных параметров. Условие на длины словарных переменных имеет вид

$$
\partial(P) \leqslant \partial(Q)
$$

где $P, Q$ - примитивные параметрические слова.

Параметрическое решение $T$ уравнения $E$ удовлетворяет условию $R$, если в результате применения преобразования $T$ к условию $R$ получается предложение, истинное для всех значений переменных.

Пусть $E$ - некоторое уравнение, возможно, с условиями, и пусть $R_{1}, \ldots, R_{m}-$ дополнительный список условий. Через $\left(E, R_{i}\right)$ мы обозначаем уравнение $E$ с дополнительным условием $R_{i}$. Будем говорить, что уравнение $E$ разделяется в список уравнений $\left(E, R_{1}\right), \ldots,\left(E, R_{m}\right)$, если каждое решение $S$ уравнения $E$ является решением некоторого уравнения $\left(E, R_{i}\right)$ из списка.

Будем говорить, что уравнение $E_{1}$ переводится параметрическим преобразованием $T$ в уравнение $E_{2}$, если $E_{1} T=E_{2}$ и для каждого решения $S_{1}$ уравнения $E_{1}$ существует решение $S_{2}$ уравнения $E_{2}$ такое, что $S_{1}=T S_{2}$. Будем говорить, что решение $S_{2}$ является образом решения $S_{1}$ при преобразовании $T$.

Пусть уравнение $E_{1}$ переводится преобразованием $T$ в уравнение $E_{2}$. Пусть $S_{1}$ - решение уравнения $E_{1}$ и $S_{2}$ - образ решения $S_{1}$ при преобразовании $T$. Если параметрическое решение $Q_{2}$ уравнения $E_{2}$ содержит решение $S_{2}$ уравнения $E_{2}$, то параметрическое решение $T Q_{2}$ уравнения $E_{1}$ содержит решение $S_{1}$ уравнения $E_{1}$.

Пусть уравнение $E_{1}$ переводится параметрическим преобразованием $T$ в уравнение $E_{2}$. Если общее решение $\left\langle E_{2}\right\rangle$ уравнения $E_{2}$ есть $Q_{1}, \ldots, Q_{r}$, то общее решение $\langle E\rangle$ уравнения $E_{1}$ есть $T Q_{1}, \ldots, T Q_{r}$.

Пусть уравнение $E$ разделяется в список уравнений $\left(E, R_{1}\right), \ldots,\left(E, R_{m}\right)$. Если общее решение $\left\langle\left(E, R_{i}\right)\right\rangle$ уравнения $\left(E, R_{i}\right)$ есть $Q_{i, 1}, \ldots, Q_{i, r_{i}}, i=1, \ldots, m$, то общее решение $\langle E\rangle$ уравнения $E$ есть $Q_{1,1}, \ldots, Q_{1, r_{1}}, \ldots, Q_{m, 1}, \ldots, Q_{m, r_{m}}$.

\section{3. Предварительные утверждения}

. Предложение 1. Общее решение уравнения

$$
x_{1} x_{2}=x_{2} x_{1}
$$


описъвается преобразованием

$$
\left\{\begin{array}{l}
x_{1} \rightarrow x_{1}^{\alpha}, \\
x_{2} \rightarrow x_{1}^{\beta},
\end{array}\right.
$$

где $\alpha, \beta$ - натуралъные параметры.

Предложение 2. Общее решение уравнения

$$
x_{1} x_{2} x_{3}=x_{3} x_{2} x_{1}
$$

описывается преобразованиями

$$
\left\{\begin{array} { l } 
{ x _ { 1 } \rightarrow x _ { 1 } ^ { \alpha } , } \\
{ x _ { 2 } \rightarrow x _ { 1 } ^ { \beta } , } \\
{ x _ { 3 } \rightarrow x _ { 1 } ^ { \gamma } , }
\end{array} \quad \left\{\begin{array}{l}
x_{1} \rightarrow\left(x_{1} x_{2}\right)^{\alpha} x_{1}, \\
x_{2} \rightarrow x_{2}\left(x_{1} x_{2}\right)^{\beta} \\
x_{3} \rightarrow\left(x_{1} x_{2}\right)^{\gamma} x_{1}
\end{array}\right.\right.
$$

где $\alpha, \beta, \gamma-$ натуралъные параметры.

Предложение 3. Общее решение уравнения

$$
x_{1}^{2} x_{2}^{2}=x_{3}^{2}
$$

описъвается преобразованиями

$$
\left\{\begin{array}{l}
x_{1} \rightarrow x_{1}^{\alpha} \\
x_{2} \rightarrow x_{1}^{\beta} \\
x_{3} \rightarrow x_{1}^{\alpha+\beta}
\end{array}\right.
$$

где $\alpha, \beta-$ натуралънъе параметры.

Доказательство. Словарные переменные удовлетворяют условию

$$
\partial\left(x_{3}\right)=\partial\left(x_{1} x_{2}\right)
$$

на длины, следовательно,

$$
x_{3}=x_{1} x_{2}
$$

Подставляя это равенство в уравнение (5), получаем $x_{1} x_{2}=x_{2} x_{1}$. Согласно предложению 1

$$
x_{1}=y^{\alpha}, \quad x_{2}=y^{\beta}
$$

где $y$ - новая словарная переменная, $\alpha, \beta$ - натуральные параметры. Используя равенство $x_{3}=x_{1} x_{2}$, получаем, что

$$
x_{3}=y^{\alpha+\beta} .
$$

Предложение 4. Общее решение уравнения

$$
x_{4} x_{3} x_{2} x_{3}=x_{1} x_{2} x_{1} x_{4}
$$


с $\partial\left(x_{4}\right) \leqslant \partial\left(x_{1}\right)$ описъвается преобразованием

$$
\left\{\begin{array}{l}
x_{1} \rightarrow x_{2}^{\beta} x_{1} \\
x_{2} \rightarrow x_{2}^{\alpha} \\
x_{3} \rightarrow x_{1} x_{2}^{\beta}, \\
x_{4} \rightarrow x_{2}^{\beta}
\end{array}\right.
$$

где $\alpha, \beta-$ натуралъные параметрь.

Доказательство. Легко видеть, что в результате применения преобразования к уравнению получается тривиальное уравнение и что преобразование удовлетворяет условию.

С другой стороны, пусть коэффициентное преобразование $S$

$$
\left\{\begin{array}{l}
x_{1} \rightarrow X_{1}, \\
x_{2} \rightarrow X_{2}, \\
x_{3} \rightarrow X_{3}, \\
x_{4} \rightarrow X_{4},
\end{array}\right.
$$

где $X_{i}, i=1, \ldots, 4,-$ слова в алфавите (1), является решением уравнения (6). По условию получаем, что

$$
X_{1}=X_{4} Y_{1}
$$

для некоторого слова $Y_{1}$. Легко видеть, что уравнение (6) переводится преобразованием $x_{1} \rightarrow x_{4} x_{1}$ в уравнение $E_{1}$

$$
x_{3} x_{2} x_{3}=x_{1} x_{2} x_{4} x_{1} x_{4},
$$

а образом решения $S$ при преобразовании $x_{1} \rightarrow x_{4} x_{1}$ будет решение $S_{1}$

$$
\left\{\begin{array}{l}
x_{1} \rightarrow Y_{1}, \\
x_{2} \rightarrow X_{2}, \\
x_{3} \rightarrow X_{3} \\
x_{4} \rightarrow X_{4}
\end{array}\right.
$$

уравнения $E_{1}$.

Словарные переменные уравнения $E_{1}$ удовлетворяют условию на длины

$$
\partial\left(x_{3}\right)=\partial\left(x_{1} x_{4}\right)
$$

следовательно,

$$
x_{3}=x_{1} x_{4} .
$$

Подставляя это равенство в уравнение $E_{1}$, получаем, что

$$
x_{4} x_{2}=x_{2} x_{4} .
$$

Согласно предложению 1

$$
x_{4}=y^{\alpha}, \quad x_{2}=y^{\beta},
$$


где $y$ - новая словарная переменная, $\alpha, \beta$ - натуральные параметры. Таким образом, общее решение уравнения (6) описывается последовательным произведением следующих преобразований:

$$
\begin{gathered}
x_{1} \rightarrow x_{4} x_{1}, \\
x_{3} \rightarrow x_{1} x_{4}, \\
\left\{\begin{array}{l}
x_{2} \rightarrow x_{2}^{\alpha}, \\
x_{4} \rightarrow x_{2}^{\beta} .
\end{array}\right.
\end{gathered}
$$

Объединяя это произведение преобразований в общее преобразование, получаем требуемое.

Предложение 5. Общее решение уравнения

$$
x_{4} x_{3} x_{2} x_{3}=x_{1} x_{2} x_{1} x_{4}
$$

$c \partial\left(x_{4}^{\lambda}\right) \leqslant \partial\left(x_{2}\right), \partial\left(x_{1} x_{2}\right) \leqslant \partial\left(x_{4}^{\lambda+1}\right) \leqslant \partial\left(x_{1}^{2} x_{2}\right)$, әде $\lambda-$ натуралъный параметр, описывается преобразованием

$$
\left\{\begin{array}{l}
x_{1} \rightarrow x_{4} x_{1}^{\alpha}, \\
x_{2} \rightarrow\left(x_{1}^{\beta} x_{4} x_{1}^{\alpha} x_{4}\right)^{\lambda} x_{1}^{\beta}, \\
x_{3} \rightarrow x_{1}^{\alpha} x_{4} \\
x_{4} \rightarrow x_{4} x_{1}^{\alpha+\beta} x_{4},
\end{array}\right.
$$

где $\alpha, \beta$ - натуралъные параметры.

Предложение 6. Общее решение уравнения

$$
x_{4} x_{2}^{2} x_{3}=x_{1} x_{3} x_{1} x_{4}
$$

c $\partial\left(\left(x_{4} x_{2}\right)^{\lambda}\right) \leqslant \partial\left(x_{3}\right), \partial\left(x_{3} x_{2}\right) \leqslant \partial\left(\left(x_{4} x_{2}\right)^{\lambda} x_{4}\right) \leqslant \partial\left(x_{3} x_{2}^{2}\right)$, әде $\lambda-$ натуралъный параметр, описывается преобразованием

$$
\left\{\begin{array}{l}
x_{1} \rightarrow x_{3}^{\beta} x_{1} \\
x_{2} \rightarrow x_{1} x_{3}^{\beta} \\
x_{3} \rightarrow\left(x_{3}^{\alpha+\beta} x_{1} x_{3}^{\beta} x_{1} x_{3}^{\beta}\right)^{\lambda} x_{3}^{\alpha} \\
x_{4} \rightarrow x_{3}^{\beta} x_{1} x_{3}^{\alpha+\beta}
\end{array}\right.
$$

где $\alpha, \beta$ - натуралъные параметры.

Предложение 7. Общее решение уравнения

$$
x_{4} x_{2}^{2} x_{3}=x_{1} x_{3} x_{1} x_{4}
$$

c $\partial\left(x_{2}\right) \leqslant \partial\left(x_{4}\right), \partial\left(\left(x_{4} x_{2}\right)^{\lambda} x_{4}\right) \leqslant \partial\left(x_{3} x_{2}\right) \leqslant \partial\left(\left(x_{4} x_{2}\right)^{\lambda+1}\right)$, где $\lambda-$ натуралъный параметр, описывается преобразованием

$$
\left\{\begin{array}{l}
x_{1} \rightarrow x_{2} x_{3}^{\alpha} \\
x_{2} \rightarrow x_{3}^{\alpha} x_{2} \\
x_{3} \rightarrow\left(x_{3}^{\alpha+\beta} x_{2} x_{3}^{\alpha} x_{2}\right)^{\lambda} x_{3}^{\alpha+\beta} \\
x_{4} \rightarrow x_{2} x_{3}^{\alpha+\beta}
\end{array}\right.
$$

где $\alpha, \beta$ - натуралъные параметры. 
Предложение 8. Общее решение уравнения

$$
x_{4} x_{3} x_{2} x_{3}=x_{1} x_{2} x_{1} x_{4}
$$

c $\partial\left(x_{1}\right) \leqslant \partial\left(x_{4}\right), \partial\left(x_{2}\right) \leqslant \partial\left(x_{4}^{\lambda+1}\right) \leqslant \partial\left(x_{1} x_{2}\right)$, где $\lambda-$ матуралъный параметр, описывается последовательным произведением преобразований

$$
\begin{aligned}
& x_{4} \rightarrow x_{1} x_{4}, \\
& x_{2} \rightarrow\left(x_{4} x_{3}\right)^{\lambda} x_{4} x_{2}, \\
& x_{3} \rightarrow x_{2} x_{3}, \\
& x_{1} \rightarrow x_{3} x_{1} .
\end{aligned}
$$

Если преобразование $T$ имеет вид

$$
\left\{\begin{array}{l}
x_{1} \rightarrow W_{1}\left(x_{1}, x_{2}, x_{3}, x_{4}\right) \\
x_{2} \rightarrow W_{2}\left(x_{1}, x_{2}, x_{3}, x_{4}\right) \\
x_{3} \rightarrow W_{3}\left(x_{1}, x_{2}, x_{3}, x_{4}\right) \\
x_{4} \rightarrow W_{4}\left(x_{1}, x_{2}, x_{3}, x_{4}\right)
\end{array}\right.
$$

то преобразование

$$
\left\{\begin{array}{l}
x_{2} \rightarrow W_{1}\left(x_{2}, x_{1}, x_{4}, x_{3}\right) \\
x_{1} \rightarrow W_{2}\left(x_{2}, x_{1}, x_{4}, x_{3}\right) \\
x_{4} \rightarrow W_{3}\left(x_{2}, x_{1}, x_{4}, x_{3}\right) \\
x_{3} \rightarrow W_{4}\left(x_{2}, x_{1}, x_{4}, x_{3}\right)
\end{array}\right.
$$

будем обозначать через $T^{\pi}$.

Предложение 9. Общее решение уравнения

$$
x_{4} x_{2}^{2} x_{3}=x_{1} x_{3} x_{1} x_{4}
$$

с $\partial\left(x_{4}\right) \leqslant \partial\left(x_{2}\right)$ описывается последовательным произведением преобразований

$$
\begin{aligned}
& x_{1} \rightarrow x_{4} x_{1}, \\
& x_{2} \rightarrow x_{1} x_{2} .
\end{aligned}
$$

Предложение 10. Общее решение уравнения

$$
x_{4} x_{2}^{2} x_{3}=x_{1} x_{3} x_{1} x_{4}
$$

c $\partial\left(x_{2}\right) \leqslant \partial\left(x_{4}\right), \partial\left(\left(x_{4} x_{2}\right)^{\lambda} x_{4}\right) \leqslant \partial\left(x_{3}\right) \leqslant \partial\left(\left(x_{4} x_{2}\right)^{\lambda+1}\right)$, где $\lambda-$ натуралънъй параметр, описывается последователъным произведением преобразований

$$
\begin{aligned}
& x_{4} \rightarrow x_{1} x_{4}, \\
& x_{3} \rightarrow\left(x_{4} x_{2}^{2}\right)^{\lambda} x_{4} x_{2} x_{3}, \\
& x_{2} \rightarrow x_{3} x_{2} \\
& x_{1} \rightarrow x_{2} x_{1} .
\end{aligned}
$$




\section{4. Параметризующие функции}

Приведем совместное индуктивное определение функций

$$
F a(y, z, v)_{1}^{\lambda_{1}, \ldots, \lambda_{s}}, \quad F a(y, z, v)_{2}^{\lambda_{1}, \ldots, \lambda_{s}},
$$

где $s \geqslant 0, y, z, v$ - словарные переменные, $\lambda_{1}, \ldots, \lambda_{s}$ - натуральные параметры:

$$
\begin{aligned}
& F a(y, z, v)_{i}=1, \quad i=1,2, \\
& F a(y, z, v)_{1}^{\lambda_{1}, \lambda_{2}, \ldots, \lambda_{s}}=z^{F a}(y, z, v)_{2}^{\lambda_{2}, \ldots, \lambda_{s}} v^{F a}(y, z, v)_{1}^{\lambda_{2}, \ldots, \lambda_{s}} \text {, } \\
& { }^{F a}(y, z, v)_{2}^{\lambda_{1}, \lambda_{2}, \ldots, \lambda_{s}}=\left({ }^{F a}(y, z, v)_{2}^{\lambda_{2}, \ldots, \lambda_{s}} v^{F a}(y, z, v)_{1}^{\lambda_{2}, \ldots, \lambda_{s}} y\right)^{\lambda_{1} F a}(y, z, v)_{2}^{\lambda_{2}, \ldots, \lambda_{s}} \text {, }
\end{aligned}
$$

в частности,

$$
\begin{aligned}
& { }^{F a}(y, z, v)_{1}^{\lambda_{1}}=z v \\
& F a(y, z, v)_{2}^{\lambda_{1}}=(v y)^{\lambda_{1}}, \\
& { }^{F a}(y, z, v)_{1}^{\lambda_{1}, \lambda_{2}}=z v(y v)^{\lambda_{2}} z v, \\
& { }^{F a}(y, z, v)_{2}^{\lambda_{1}, \lambda_{2}}=\left((v y)^{\lambda_{2}} v z v y\right)^{\lambda_{1}}(v y)^{\lambda_{2}} .
\end{aligned}
$$

Рассмотрим уравнение вида

$$
x_{1} P x_{2} U=Q x_{2} R x_{1} V
$$

где $P, Q, R$ - примитивные параметрические слова в алфавите $\left\{x_{3}, \ldots, x_{n}\right\}, U, V-$ параметрические слова. Рассмотрим последовательность параметрических преобразований

$$
\begin{gathered}
T_{1}=\left\{\begin{array}{l}
x_{1} \rightarrow Q x_{2} R x_{1}, \\
x_{2} \rightarrow\left(x_{2} R x_{1} P\right)^{\lambda_{1}} x_{2},
\end{array}\right. \\
\cdots \\
T_{k}=\left\{\begin{array}{l}
x_{1} \rightarrow Q x_{2} R x_{1}, \\
x_{2} \rightarrow\left(x_{2} R x_{1} P\right)^{\lambda_{k}} x_{2},
\end{array}\right.
\end{gathered}
$$

где $\lambda_{1}, \ldots, \lambda_{k}, \ldots$ - натуральные параметры.

Теорема 1. Для каждого натуралъного s произведение первых s параметрических преобразований из последовательности преобразований (13) совпадает с преобразованием

$$
\left\{\begin{array}{l}
x_{1} \rightarrow^{F a}\left(x_{1} P, Q, x_{2} R\right)_{1}^{\lambda_{1}, \ldots, \lambda_{s}} x_{1}, \\
x_{2} \rightarrow^{F a}\left(x_{1} P, Q, x_{2} R\right)_{2}^{\lambda_{1}, \ldots, \lambda_{s}} x_{2} .
\end{array}\right.
$$

Доказательство. При $s=0$ теорема очевидна. Пусть $s>0$. Предположим, что произведение преобразований $T_{2}, \ldots, T_{s}$ совпадает с преобразованием

$$
\left\{\begin{array}{l}
x_{1} \rightarrow^{F a}\left(x_{1} P, Q, x_{2} R\right)_{1}^{\lambda_{2}, \ldots, \lambda_{s}} x_{1}, \\
x_{2} \rightarrow^{F a}\left(x_{1} P, Q, x_{2} R\right)_{2}^{\lambda_{2}, \ldots, \lambda_{s}} x_{2}
\end{array}\right.
$$


По условию первое преобразование имеет вид

$$
\left\{\begin{array}{l}
x_{1} \rightarrow Q x_{2} R x_{1}, \\
x_{2} \rightarrow\left(x_{2} R x_{1} P\right)^{\lambda_{1}} x_{1} .
\end{array}\right.
$$

Следовательно, произведение первых $s$ преобразований совпадает с преобразованием

$$
\left\{\begin{aligned}
x_{1} \rightarrow & Q^{F a}\left(x_{1} P, Q, x_{2} R\right)_{2}^{\lambda_{2}, \ldots, \lambda_{s}} x_{2} R^{F a}\left(x_{1} P, Q, x_{2} R\right)_{1}^{\lambda_{2}, \ldots, \lambda_{s}} x_{1}, \\
x_{2} \rightarrow & \left({ }^{F a}\left(x_{1} P, Q, x_{2} R\right)_{2}^{\lambda_{2}, \ldots, \lambda_{s}} x_{2} R^{F a}\left(x_{1} P, Q, x_{2} R\right)_{1}^{\lambda_{2}, \ldots, \lambda_{s}} x_{1} P\right)^{\lambda_{1}} \\
& \times{ }^{F a}\left(x_{1} P, Q, x_{2} R\right)_{2}^{\lambda_{2}, \ldots, \lambda_{s}} x_{2} .
\end{aligned}\right.
$$

Теперь остается воспользоваться определением функций $F a(y, z, v)_{1}^{\lambda_{1}, \ldots, \lambda_{s}}$ и $F a(y, z, v)_{2}^{\lambda_{1}, \ldots, \lambda_{s}}$.

Пусть

$$
\mu_{1}, \mu_{2}, \ldots, \mu_{u}, \ldots
$$

- счетный алфавит переменных, значениями которых являются конечные последовательности натуральных параметров.

Предложение 11. Общее решение уравнения

$$
x_{4} x_{3} x_{2} x_{3}=x_{1} x_{2} x_{1} x_{4}
$$

описывается преобразованиями

$$
{ }^{F c} 1(\mu)\langle 6\rangle, \quad{ }^{F a} 1(\mu)\langle 7\rangle, \quad{ }^{F a} 1(\mu)\langle 10\rangle
$$

¿de

$$
{ }^{F a} 1(\mu)=\left\{\begin{array}{l}
x_{4} \rightarrow{ }^{F a}\left(x_{4} x_{3}, x_{1}, x_{2} x_{1}\right)_{1}^{\mu} x_{4}, \\
x_{2} \rightarrow F a\left(x_{4} x_{3}, x_{1}, x_{2} x_{1}\right)_{2}^{\mu} x_{2},
\end{array}\right.
$$

а $\mu$ - переменная для последовательностей натуральных параметров.

Доказателъство. Пользуясь определением функций

$$
F a\left(x_{1}, x_{2}, x_{3}\right)_{i}^{\lambda_{1}, \lambda_{2}, \ldots, \lambda_{s}}, \quad s \geqslant 0, \quad i=1,2,
$$

легко убедиться, что в результате применения преобразования $F a 1(\mu)$ к уравнению (14) вновь получается уравнение (14) и, следовательно, каждое из трех преобразований предложения 11 является параметрическим решением уравнения (14).

С другой стороны, пусть коэффициентное преобразование $S$

$$
\left\{\begin{array}{l}
x_{1} \rightarrow X_{1} \\
x_{2} \rightarrow X_{2} \\
x_{3} \rightarrow X_{3} \\
x_{4} \rightarrow X_{4}
\end{array}\right.
$$

где $X_{i}, i=1, \ldots, 4,-$ слова в алфавите (1), есть произвольное решение уравнения (14).

Индукцией по $\left|X_{1} X_{2} X_{3} X_{4}\right|$ мы докажем, что преобразование $S$ содержится в списке параметрических преобразований предложения 11.

Легко видеть, что найдется натуральное число $t$ такое, что длины компонент решения $S$ будут удовлетворять хотя бы одному из следующих четырех условий: 
(1) $\left|X_{4}\right| \leqslant\left|X_{1}\right|$

(2) $\left|X_{1}\right| \leqslant\left|X_{4}\right|,\left|X_{4}^{t}\right| \leqslant\left|X_{2}\right| \leqslant\left|X_{4}^{t+1}\right|,\left|X_{4}^{t+1}\right| \leqslant\left|X_{1} X_{2}\right|$,

(3) $\left|X_{1}\right| \leqslant\left|X_{4}\right|,\left|X_{4}^{t}\right| \leqslant\left|X_{2}\right| \leqslant\left|X_{4}^{t+1}\right|,\left|X_{1} X_{2}\right| \leqslant\left|X_{4}^{t+1}\right| \leqslant\left|X_{1}^{2} X_{2}\right|$,

(4) $\left|X_{1}\right| \leqslant\left|X_{4}\right|,\left|X_{4}^{t}\right| \leqslant\left|X_{2}\right| \leqslant\left|X_{4}^{t+1}\right|,\left|X_{1}^{2} X_{2}\right| \leqslant\left|X_{4}^{t+1}\right|$.

Если компоненты решения $S$ удовлетворяют условию 1 , то решение $S$ содержится в параметрическом преобразовании ${ }^{F a} 1(\mu)\langle 6\rangle$ при пустом значении для $\mu$.

Если компоненты решения $S$ удовлетворяют условию 2 , то решение $S$ содержится в параметрическом преобразовании ${ }^{F a} 1(\mu)\langle 10\rangle$ при пустом значении для $\mu$.

Если компоненты решения $S$ удовлетворяют условию 3 , то решение $S$ содержится в параметрическом преобразовании ${ }^{F a} 1(\mu)\langle 7\rangle$ при пустом значении для $\mu$.

Пусть длины компонент решения $S$ удовлетворяют условию 4 . Тогда

$$
X_{4}=X_{1} Y_{4}
$$

для некоторого слова $Y_{4}$. Легко видеть, что уравнение (14) переводится преобразованием

$$
x_{4} \rightarrow x_{1} x_{4}
$$

в уравнение $E_{1}$

$$
x_{4} x_{3} x_{2} x_{3}=x_{2} x_{1}^{2} x_{4}
$$

а образом решения $S$ при преобразовании $x_{4} \rightarrow x_{1} x_{4}$ будет решение $S_{1}$

$$
\left\{\begin{array}{l}
x_{1} \rightarrow X_{1}, \\
x_{2} \rightarrow X_{2}, \\
x_{3} \rightarrow X_{3}, \\
x_{4} \rightarrow Y_{4}
\end{array}\right.
$$

уравнения $E_{1}$. Длины компонент решения $S_{1}$ будут удовлетворять условиям

$$
\begin{gathered}
\left|\left(X_{1} Y_{4}\right)^{t}\right| \leqslant\left|X_{2}\right| \leqslant\left|\left(X_{1} Y_{4}\right)^{t+1}\right| \\
\left|X_{1}^{2} X_{2}\right| \leqslant\left|\left(X_{1} Y_{4}\right)^{t+1}\right|, \\
\left|X_{1}\right|=\left|X_{3}\right|
\end{gathered}
$$

и, следовательно, $X_{2}=\left(Y_{4} X_{3}\right)^{t} Y_{2}$ для некоторого слова $Y_{2}$. Легко видеть, что уравнение $E_{1}$ переводится преобразованием

$$
x_{2} \rightarrow\left(x_{4} x_{3}\right)^{\lambda} x_{2}
$$

где $\lambda$ - натуральный параметр, в уравнение $E_{2}$

$$
x_{4} x_{3} x_{2} x_{3}=x_{2} x_{1}^{2} x_{4}
$$

с $\partial\left(x_{2}\right) \leqslant \partial\left(x_{4} x_{3}\right)$, а образом решения $S_{1}$ при преобразовании

$$
x_{2} \rightarrow\left(x_{4} x_{3}\right)^{\lambda} x_{2}
$$


будет решение $S_{2}$

$$
\left\{\begin{array}{l}
x_{1} \rightarrow X_{1}, \\
x_{2} \rightarrow Y_{2}, \\
x_{3} \rightarrow X_{3}, \\
x_{4} \rightarrow Y_{4}
\end{array}\right.
$$

уравнения $E_{2}$. Длины компонент решения $S_{2}$ будут удовлетворять условиям

$$
\left|X_{1}\right|=\left|X_{3}\right|, \quad\left|X_{1} Y_{2}\right| \leqslant\left|Y_{4}\right|,
$$

и, следовательно,

$$
Y_{4}=X_{2} X_{1} Z_{4}
$$

для некоторого слова $Z_{4}$. Легко видеть, что уравнение $E_{2}$ переводится преобразованием

$$
x_{4} \rightarrow x_{2} x_{1} x_{4}
$$

в уравнение $E_{3}$

$$
x_{4} x_{3} x_{2} x_{3}=x_{1} x_{2} x_{1} x_{4},
$$

а образом решения $S_{2}$ при преобразовании $x_{4} \rightarrow x_{2} x_{1} x_{4}$ будет решение $S_{3}$

$$
\left\{\begin{array}{l}
x_{1} \rightarrow X_{1}, \\
x_{2} \rightarrow Y_{2}, \\
x_{3} \rightarrow X_{3} \\
x_{4} \rightarrow Z_{4}
\end{array}\right.
$$

уравнения $E_{3}$.

Уравнение $E_{3}$ совпадает с уравнением (14) и

$$
\left|X_{1} Y_{2} X_{3} Z_{4}\right|<\left|X_{1} X_{2} X_{3} X_{4}\right| \text {. }
$$

По индуктивному предположению решение $S_{3}$ содержится в списке

$$
{ }^{F a} 1(\mu)\langle 6\rangle, \quad F a 1(\mu)\langle 7\rangle, \quad{ }^{F a} 1(\mu)\langle 10\rangle .
$$

Следовательно, исходное решение $S$ содержится в списке

$$
T^{F a} 1(\mu)\langle 6\rangle, \quad T^{F a} 1(\mu)\langle 7\rangle, \quad T^{F a} 1(\mu)\langle 10\rangle,
$$

где $T$ есть последовательное произведение преобразований

$$
\begin{aligned}
& x_{4} \rightarrow x_{1} x_{4}, \\
& x_{2} \rightarrow\left(x_{4} x_{3}\right)^{\lambda} x_{2}, \\
& x_{4} \rightarrow x_{2} x_{1} x_{4},
\end{aligned}
$$

то есть преобразование

$$
\left\{\begin{array}{l}
x_{4} \rightarrow x_{1} x_{2} x_{1} x_{4}, \\
x_{2} \rightarrow\left(x_{2} x_{1} x_{4} x_{3}\right)^{\lambda} x_{2} .
\end{array}\right.
$$

Используя теорему 1 , легко доказать, что

$$
T^{F a} 1(\mu)={ }^{F a} 1(\lambda, \mu) .
$$


Предложение 12. Общее решение уравнения

$$
x_{4} x_{2}^{2} x_{3}=x_{1} x_{3} x_{1} x_{4}
$$

описывается преобразованиями

$$
{ }^{F a} 2(\mu)\langle 11\rangle, \quad{ }^{F a} 2(\mu)\langle 8\rangle, \quad{ }^{F a} 2(\mu)\langle 9\rangle, \quad{ }^{F a} 2(\mu)\langle 12\rangle,
$$

гдe

$$
F a 2(\mu)=\left\{\begin{array}{l}
x_{4} \rightarrow^{F a}\left(x_{4} x_{2}^{2}, x_{1}, x_{3} x_{1}\right)_{1}^{\mu} x_{4}, \\
x_{3} \rightarrow^{F a}\left(x_{4} x_{2}^{2}, x_{1}, x_{3} x_{1}\right)_{2}^{\mu} x_{3},
\end{array}\right.
$$

а $\mu$ - переменная для последовательностей натуралъных параметров.

Введем обозначения для примитивно параметрических преобразований:

$$
\begin{aligned}
& A(\lambda)=\left\{\begin{array}{l}
x_{1} \rightarrow x_{3} x_{1}, \\
x_{2} \rightarrow\left(x_{4} x_{2} x_{3}\right)^{\lambda} x_{4} x_{2}, \\
x_{3} \rightarrow x_{2} x_{3}, \\
x_{4} \rightarrow x_{3} x_{1} x_{4},
\end{array}\right. \\
& B(\lambda)=\left\{\begin{array}{l}
x_{1} \rightarrow x_{2} x_{1}, \\
x_{2} \rightarrow x_{3} x_{2}, \\
x_{3} \rightarrow\left(x_{4}\left(x_{3} x_{2}\right)^{2}\right)^{\lambda} x_{4} x_{3} x_{2} x_{3}, \\
x_{4} \rightarrow x_{2} x_{1} x_{4},
\end{array}\right. \\
& C(\lambda)=\left\{\begin{array}{l}
x_{1} \rightarrow x_{4} x_{1}, \\
x_{2} \rightarrow x_{1} x_{2},
\end{array}\right.
\end{aligned}
$$

где $\lambda$ - натуральный параметр.

Преобразование

$$
{ }^{L a}\left(\mu_{1}, \lambda_{1}, \mu_{2}, \lambda_{2}, \ldots, \mu_{2 k-1}, \lambda_{2 k-1}, \mu_{2 k}, \lambda_{2 k}\right),
$$

где $k \geqslant 0, \mu_{1}, \mu_{2}, \ldots, \mu_{2 k-1}, \mu_{2 k}$ - переменные для последовательностей натуральных параметров, $\lambda_{1}, \lambda_{2}, \ldots, \lambda_{2 k-1}, \lambda_{2 k}-$ натуральные параметры, определяемые следующим произведением преобразований

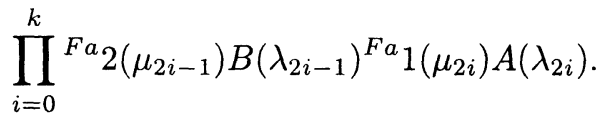

Пусть

$$
\nu_{1}, \nu_{2}, \ldots, \nu_{v}, \ldots
$$

- счетный алфавит переменных, значениями которых являются последовательности вида

$$
\mu_{1}, \lambda_{1}, \mu_{2}, \lambda_{2}, \ldots, \mu_{2 k-1}, \lambda_{2 k-1}, \mu_{2 k}, \lambda_{2 k},
$$

где $\mu_{i}$ - переменные для последовательностей натуральных параметров, $\lambda_{i}$ - натуральные параметры.

Через $^{L a}(\nu)$ будем обозначать параметризующую функцию, зависящую от параметра $\nu$. 
Предложение 13. Общее решение уравнения

$$
x_{4} x_{2}^{2} x_{3}=x_{1} x_{3} x_{1} x_{4}
$$

описывается преобразованиями

$$
\begin{aligned}
& { }^{L a}(\nu)\langle 8\rangle, \\
& { }^{L a}(\nu)\langle 9\rangle, \\
& { }^{L a}(\nu)^{F a} 2(\mu) B(\lambda)\langle 6\rangle, \\
& { }^{L a}(\nu)^{F a} 2(\mu) B(\lambda)\langle 7\rangle, \\
& { }^{L a}(\nu)^{F a} 2(\mu) C^{F a} 1\left(\mu_{2}\right)^{\pi} A(\lambda)^{\pi}\langle 16\rangle^{\pi},
\end{aligned}
$$

где $\nu$ - переменная для последователъностей вида

$$
\mu_{1}, \lambda_{1}, \mu_{2}, \lambda_{2}, \ldots, \mu_{2 k-1}, \lambda_{2 k-1}, \mu_{2 k}, \lambda_{2 k} .
$$

Преобразование

$$
\begin{aligned}
&{ }^{D o}\left(\nu_{1}, \mu_{1}, \mu_{2}, \lambda_{1}, \nu_{2}, \mu_{3}, \mu_{4}, \lambda_{2}, \ldots,\right. \\
&\left.\nu_{2 k-1}, \mu_{4 k-3}, \mu_{4 k-2}, \lambda_{2 k-1}, \nu_{2 k}, \mu_{4 k-1}, \mu_{4 k}, \dot{\lambda}_{2 k}\right), \quad \quad k \geqslant 0,
\end{aligned}
$$

определяется следующим произведением преобразований:

$$
\prod_{i=0}^{k} L a\left(\nu_{2 i-1}\right)^{F a} 2\left(\mu_{4 i-3}\right) C^{F a} 1\left(\mu_{4 i-2}\right)^{\pi} A\left(\lambda_{2 i-1}\right)^{\pi L a}\left(\nu_{2 i}\right)^{\pi F a} 2\left(\mu_{4 i-1}\right)^{\pi} C^{\pi F a} 1\left(\mu_{4 i}\right) A\left(\lambda_{2 i}\right) .
$$

Пусть

$$
\xi_{1}, \xi_{2}, \ldots, \xi_{w}, \ldots
$$

- счетный алфавит переменных, значениями которых являются последовательности вида

$$
\nu_{1}, \mu_{1}, \mu_{2}, \lambda_{1}, \nu_{2}, \mu_{3}, \mu_{4}, \lambda_{2}, \ldots, \nu_{2 k-1}, \mu_{4 k-3}, \mu_{4 k-2}, \lambda_{2 k-1}, \nu_{2 k}, \mu_{4 k-1}, \mu_{4 k}, \lambda_{2 k}, \quad k \geqslant 0 \text {. }
$$

Через ${ }^{D o}(\xi)$ обозначаем параметризующую функию, зависящую от параметра $\xi$. Предложение 14. Общее решение уравнения

$$
x_{4} x_{2}^{2} x_{3}=x_{1} x_{3} x_{1} x_{4}
$$

описывается преобразованиями

$$
\begin{aligned}
& { }^{D o}(\xi)^{L a}(\nu)\langle 8\rangle \\
& { }^{D o}(\xi)^{L a}(\nu)\langle 9\rangle \\
& { }^{D o}(\xi)^{L a}(\nu)^{F a} 2(\mu) B(\lambda)\langle 6\rangle, \\
& { }^{D o}(\xi)^{L a}(\nu)^{F a} 2(\mu) B(\lambda)\langle 7\rangle, \\
& { }^{D o}(\xi)^{L a}\left(\nu_{1}\right)^{F a} 2\left(\mu_{1}\right) C\left\langle 6^{\pi}\right\rangle, \\
& { }^{D o}(\xi)^{L a}\left(\nu_{1}\right)^{F a} 2\left(\mu_{1}\right) C\left\langle 7^{\pi}\right\rangle, \\
& { }^{D o}(\xi)^{L a}\left(\nu_{1}\right)^{F a} 2\left(\mu_{1}\right) C^{F a} 1\left(\mu_{2}\right)^{\pi} A\left(\lambda_{1}\right)^{\pi L a}\left(\nu_{1}\right)^{\pi F a} 2\left(\mu_{1}\right) C^{\pi}\langle 6\rangle, \\
& { }^{D o}(\xi)^{L a}\left(\nu_{1}\right)^{F a} 2\left(\mu_{1}\right) C^{F a} 1\left(\mu_{2}\right)^{\pi} A\left(\lambda_{1}\right)^{\pi L a}\left(\nu_{1}\right)^{\pi F a} 2\left(\mu_{1}\right) C^{\pi}\langle 7\rangle,
\end{aligned}
$$


әде $\xi$ - переменная для последовательностей вида

$$
\nu_{1}, \mu_{1}, \mu_{2}, \lambda_{1}, \nu_{2}, \mu_{3}, \mu_{4}, \lambda_{2}, \ldots, \nu_{2 k-1}, \mu_{4 k-3}, \mu_{4 k-2}, \lambda_{2 k-1}, \nu_{2 k}, \mu_{4 k-1}, \mu_{4 k}, \lambda_{2 k}
$$

Предложение 15. Общее решение уравнения

$$
x_{1}^{2} x_{2} x_{4}=x_{4} x_{3} x_{2} x_{3}
$$

описывается преобразованиями

$$
\begin{gathered}
x_{4} \rightarrow x_{1} x_{4}, \\
\langle 14\rangle, \\
\left\{\begin{array}{l}
x_{1} \rightarrow x_{4} x_{1}^{\alpha}, \\
x_{2} \rightarrow x_{1}^{\beta} \\
x_{3} \rightarrow x_{1}^{\alpha} x_{4},
\end{array}\right.
\end{gathered}
$$

где $\alpha, \beta$ - натуралъные параметръ.

Предложение 16. Общее решение уравнения

$$
x_{2} x_{1}^{2} x_{4}=x_{3} x_{4} x_{2} x_{3}
$$

описывается преобразованиями

$$
\begin{array}{ll}
x_{2} \rightarrow\left(x_{3} x_{4}\right)^{\lambda} x_{2}, & x_{2} \rightarrow\left(x_{3} x_{4}\right)^{\lambda} x_{2}, \\
x_{3} \rightarrow x_{2} x_{3}, & x_{2} \rightarrow x_{3} x_{2}, \\
x_{1} \rightarrow x_{3} x_{2}, & x_{4} \rightarrow x_{2} x_{4},
\end{array}
$$

$\langle 15\rangle$,

Предложение 17. Общее решение уравнения

$$
x_{1}^{2} x_{2}^{2} x_{3}^{2}=x_{4}^{2}
$$

описывается преобразованием

$$
\begin{gathered}
x_{4} \rightarrow x_{1} x_{4} x_{3}, \\
\left\langle 19^{\pi}\right\rangle .
\end{gathered}
$$

Предложение 18. Общее решение уравнения

$$
x_{1}^{2} x_{2}^{2}=x_{3}^{2} x_{4}^{2}
$$

описъвается преобразованием

$$
\left\{\begin{array}{l}
x_{1} \rightarrow\left(x_{2} x_{1}\right)^{(\alpha+\beta+1) \lambda+\alpha} x_{2}\left(x_{2} x_{1}\right)^{\alpha+\beta+1} \\
x_{2} \rightarrow\left(x_{2} x_{1}\right)^{\gamma} x_{2} \\
x_{3} \rightarrow\left(x_{2} x_{1}\right)^{(\alpha+\beta+1) \lambda+\alpha} x_{2} \\
x_{4} \rightarrow\left(x_{1} x_{2}\right)^{\alpha+\beta+1}\left(x_{2} x_{1}\right)^{\gamma} x_{2}
\end{array}\right.
$$

где $\alpha, \beta, \gamma, \lambda$ - натуралъные параметры. 


\section{Список литературы}

1. Хмелевский Ю. И., Решение уравнений в словах с тремя неизвестными. Докл. АН СССР (1967) 177, 1023-1025.

2. Хмелевский Ю. И., Уравнения в свободной полугруппе. Трудъ МИАН (1971) 107, 281284.

3. Makanin G. S., On the general solution of quations in a free semigroup. Lect. Notes Computer Sci. (1991) 677, 1-5.

4. Makanin G. S., Multiplication of natural number parameters and equations in a free semigroup. Trans. Amer. Math. Soc. (1996) 348, 4813-4824.

5. Makanin G. S., Abdulrab H., Goralcic P., Functions for the general solution of parametric Word equations. Lect. Notes Computer Sci. (1997) 1234, 189-202.

Статья поступила 14.05.1998. 